\title{
Direct Observation of Double-Core-Hole Shake-Up States in Photoemission
}

\author{
Ralph Püttner, ${ }^{1}$ Gildas Goldsztejn, ${ }^{2,3}$ Denis Céolin, ${ }^{4}$ Jean-Pascal Rueff, ${ }^{4}$ Thierry Moreno, ${ }^{4}$ \\ Rajesh K. Kushawaha, ${ }^{2,3}$ Tatiana Marchenko, ${ }^{2,3}$ Renaud Guillemin,, ${ }^{2,3}$ Loïc Journel, ${ }^{2,3}$ \\ Dennis W. Lindle, ${ }^{5}$ Maria Novella Piancastelli, ${ }^{2,3,6}$ and Marc Simon ${ }^{2,3,4}$ \\ ${ }^{1}$ Institut für Experimentalphysik, Freie Universität Berlin, Arnimallee 14, D-14195 Berlin, Germany \\ ${ }^{2}$ CNRS, UMR 7614, Laboratoire de Chimie Physique-Matière et Rayonnement, F-75005 Paris, France \\ ${ }^{3}$ Sorbonne Universités, UPMC Univ Paris 06, UMR 7614, Laboratoire de Chimie Physique-Matière et Rayonnement, \\ F-75005 Paris, France \\ ${ }^{4}$ Synchrotron SOLEIL, l'Orme des Merisiers, Saint-Aubin, F-91192 Gif-sur-Yvette Cedex, France \\ ${ }^{5}$ Department of Chemistry, University of Nevada, Las Vegas, 4505 South Maryland Parkway, \\ P.O. Box 4003, Las Vegas, Nevada 89154-4003, USA \\ ${ }^{6}$ Department of Physics and Astronomy, Uppsala University, SE-75120 Uppsala, Sweden
}

(Received 8 October 2014; published 4 March 2015)

Direct measurements of $\mathrm{Ar}^{+} 1 s^{-1} 2 p^{-1} n l$ double-core-hole shake-up states are reported using conventional single-channel photoemission, offering a new and relatively easy means to study such species. The high-quality results yield accurate energies and lifetimes of the double-core-hole states. Their photoemission spectrum also can be likened to $1 s$ absorption of an exotic argon ion with a $2 p$ core vacancy, providing new information about the spectroscopy of both this unusual ionic state as well as the neutral atom.

DOI: 10.1103/PhysRevLett.114.093001

In recent years, studies of double-core-hole (DCH) states in molecules have attracted much attention [1-8]. This interest stems in part because the large chemical shifts in DCH states may be combined with the smaller, but wellknown, electron spectroscopy for chemical analysis (ESCA) [9] chemical shifts, derived from single-core photoemission, to differentiate the effects of relaxation from the influence of the chemical environment in a molecule [10]. However, to date, observations of DCH states have often required heroic experimental efforts. Indeed, the most recent experiments either resorted to complex multielectron coincidence techniques using synchrotron radiation (SR) [1-5] or they involved complicated multiphoton absorption processes using an $\mathrm{x}$-ray freeelectron laser [6,7].

It is the purpose of this work to demonstrate, using argon as a prototype, that DCH states may be studied readily via standard single-channel photoelectron spectroscopy using SR. Consider photoemission leading to the $\mathrm{Ar}^{+} 1 s^{-1} 2 p^{-1} n p$ $(n \geq 4)$ states. These are single-ion states, so only one photoelectron is emitted, producing what can be described as a core shake-up (satellite) state: $1 s$ photoionization accompanied by shakeup of a core $2 p$ electron to the $n p$ Rydberg orbital. If an entire Rydberg series can be measured, double-core ionization thresholds can be determined accurately. Furthermore, if the $1 s$ and $2 p$ core holes are weakly coupled, the $1 s^{-1} 2 p^{-1} n p$ Rydberg series observed in photoemission can be viewed as the $1 s$ near-edge $\mathrm{x}$-ray-absorption fine-structure (NEXAFS) spectrum of an $\mathrm{Ar}^{+}$ion with a vacancy in the $2 p$ subshell. All of this is now feasible using photoemission, as is demonstrated below.
PACS numbers: 32.80.Aa, 32.30.Rj, 32.70.Jz, 32.80.Ee

Satellite, or shake-up, processes in the photoionization of atoms and molecules, of course, have been studied for several decades; see, e.g., Refs. [11-13]. A thorough discussion on such processes in rare gases is reported in Ref. [14]. However, until now, direct observations of satellites in conventional photoelectron spectroscopy have been limited to cases where only inner or outer valence electrons are involved in the shake-up process. Core-hole photoemission satellites (i.e., DCH states) have been observed only indirectly using photoabsorption $[15,16]$ or x-ray emission $[17,18]$, in coincidence studies [2-4], or in multiphoton experiments [6,7]. Specifically for argon, three early photoabsorption studies focused on the $1 s^{-1} 2 l^{-1} 4 p^{2}$ doubly excited states $[15,16,19]$. One of them [16] derived approximate binding energies for the $1 s^{-1} 2 p^{-1} n l$ core shake-up satellites as well as $1 s^{-1} 2 p^{-1}$ $\mathrm{DCH}$ ionization thresholds. A few years later, $\mathrm{Ar}$ $1 s^{-1} 2 l^{-1} n l$ shake-up satellites were studied using $K L$ $\mathrm{x}$-ray emission $[17,18]$, yielding shake-up probabilities for these states. The latter work [18] also reported approximate values for the $1 s^{-1} 2 s^{-1} \mathrm{DCH}$ thresholds. A very recent $K L$ $\mathrm{x}$-ray emission study focused on the $1 s^{-1} 2 p^{-1} n l n^{\prime} l^{\prime}$ doubly excited states [20], revealing a higher fluorescence rate for the $1 s^{-1} 2 p^{-1}\left({ }^{1} P\right)$ states than for the $1 s^{-1} 2 p^{-1}\left({ }^{3} P\right)$ states.

In the present study, $\mathrm{x}$-ray photoelectron spectra of $\mathrm{Ar}^{+}$ $1 s^{-1} 2 p^{-1} n l$ DCH shake-up states are reported. Several Rydberg series converging to the $1 s^{-1} 2 p^{-1}$ thresholds are visible in the spectra, permitting derivation of accurate energies for the DCH thresholds. The quality of the spectra also allows accurate determination of the lifetimes of the DCH satellite states, resulting in a value comparable to that 
of the Ar $1 s$ singly core-ionized state. This result, coupled to a comparison between the Ar $1 s$ NEXAFS spectrum for the neutral (ground-state) atom and the photoemission spectrum of the DCH states (a pseudo- $1 s$ NEXAFS spectrum of argon with a $2 p$ hole) is shown to lead to previously unavailable information about the neutral-atom spectrum.

To accomplish this study, an efficient experimental setup that includes a high-brightness, high-resolution $\mathrm{x}$-ray source coupled to high-throughput, high-resolution electron analysis was required. The measurements were performed at the SOLEIL Synchrotron, France, on the GALAXIES beam line, with a newly operational end station dedicated to hard-x-ray photoelectron spectroscopy (HAXPES) [21,22]. Linearly polarized light was provided by a U20 undulator and energy selected by a $\mathrm{Si}(111)$ double-crystal monochromator. Photoelectron spectra were collected with a Scienta EW4000 analyzer specifically designed for HAXPES. To obtain accurate binding energies from the photoelectron spectra, energy calibration was achieved in two steps. First, the kinetic-energy scale of the electron analyzer was calibrated by measuring $\mathrm{Ar}$ $K M M$ and $L M M$ Auger spectra. The Auger energies were calculated from binding energies of 3206.3(3) eV [23], 248.63(1) eV [24,25], and 45.14(1) eV [26] for the $1 s^{-1}$, $2 p_{3 / 2}^{-1}$, and $3 p^{-2}\left({ }^{1} D_{2}\right)$ states of argon, respectively. Second, the photon energy was calibrated by measuring $\mathrm{Ar}^{-1} s^{-1}$ and $2 p_{3 / 2}^{-1}$ photoelectron spectra using the previously calibrated kinetic-energy scale. Overall, the binding energies for the $\mathrm{DCH}$ states were determined with a systematic uncertainty of $0.4 \mathrm{eV}$. The accuracy of the binding energies is limited mostly by uncertainty in the literature value of the $1 s^{-1}$ binding energy.

Figure 1 shows a photoelectron spectrum in the bindingenergy region of the $\operatorname{Ar} 1 s^{-1} 2 l^{-1}$ shake-up satellites. The portion below $3520 \mathrm{eV}$ binding energy is dominated by

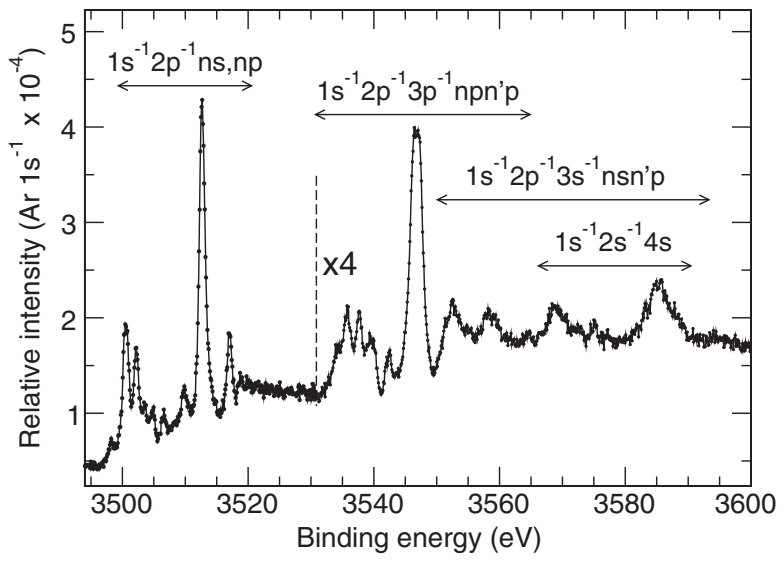

FIG. 1. The $1 s^{-1} 2 l^{-1}$ DCH shake-up photoelectron spectrum of argon, relative to the intensity of $\operatorname{Ar} 1 s^{-1}$ photoionization. Above $3530 \mathrm{eV}$, the relative intensity is multiplied by a factor of 4 .
$1 s^{-1} 2 p^{-1} n s, n p$ satellites converging to the $1 s^{-1} 2 p^{-1}\left({ }^{1,3} P\right)$ double-ionization thresholds. At higher binding energies, $1 s^{-1} 2 p^{-1} 3 p^{-1} n p n^{\prime} p$ and $1 s^{-1} 2 p^{-1} 3 s^{-1} n s n^{\prime} p$ double shake-up satellites are visible, overlapping somewhat with $1 s^{-1} 2 s^{-1} 4 s$ satellites above $3565 \mathrm{eV}$ binding energy. The $1 s^{-1} 2 p^{-1} n l$ region of the spectrum, below $3530 \mathrm{eV}$ binding energy, was collected using a photon energy of $\mathrm{h} \nu=3900 \mathrm{eV}$, while the higher-binding-energy region was collected with $\mathrm{h} \nu=3970 \mathrm{eV}$. Here we concentrate on the $1 s^{-1} 2 p^{-1} n l$ photoelectron satellites at lower energy. The remainder of the spectrum will be dealt with in a future publication.

An expanded spectrum of the $\operatorname{Ar} 1 s^{-1} 2 p^{-1} n l$ DCH satellite region is shown in Fig. 2, along with the results of a fit (solid curve). To fit the spectra, all of the photoelectron peaks were simulated with postcollisioninteraction line shapes using the formula of Armen et al. [27]. The peaks were convoluted with a Gaussian to account for the finite experimental resolution, and both, one lifetime broadening $\Gamma$ used for all Rydberg states and the Gaussian width $\Delta$, were left as free parameters in the analysis. Note the fit properly accounts for the fact that the $\operatorname{Ar} 1 s^{-1} 2 p^{-1} \mathrm{DCH}$ configuration splits into four states, ${ }^{3} P_{2,1,0}$ and ${ }^{1} P_{1}$, with different binding energies. The background is composed of a linear function as well as an arctan function for each of the four thresholds to account for unresolved Rydberg states and continuum contributions. From the fit in Fig. 2, $\Gamma$ was determined to be 675 (20) $\mathrm{meV}$ FWHM, and $\Delta$ was found to be $525(20) \mathrm{meV}$ FWHM. The latter value compares well with an ideal value of $500 \mathrm{meV}$ expected under the experimental conditions, based on $476 \mathrm{meV}$ FWHM for the photon bandwidth, obtained by ray-tracing calculations using the actual

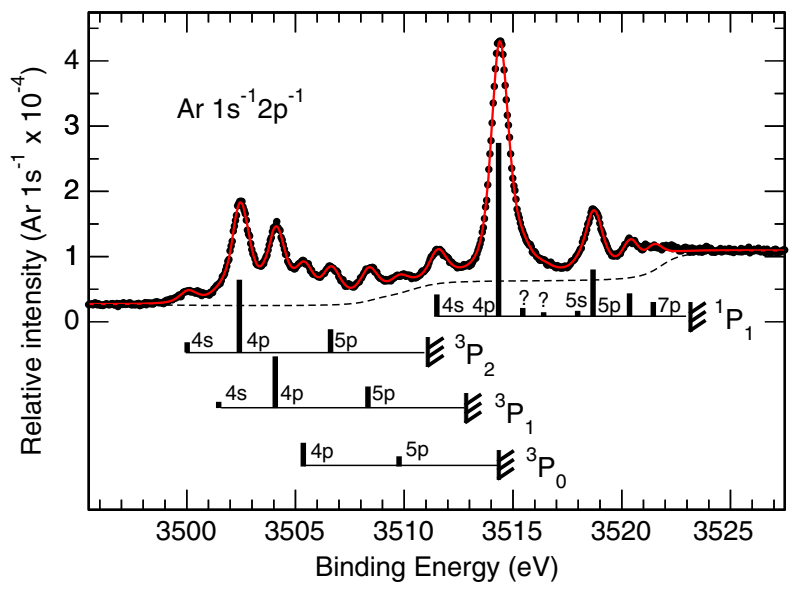

FIG. 2 (color online). The $1 s^{-1} 2 p^{-1}$ DCH shake-up photoelectron spectrum of argon, relative to the intensity of $\mathrm{Ar} 1 s^{-1}$ photoionization. The solid curve through the data points is a fit, and the dashed curve represents the background (see text for details). The bar diagrams indicate positions and intensities of Rydberg series converging to the four different ionization thresholds. 
experimental settings, $125 \mathrm{meV}$ FWHM for the spectrometer resolution, and $80 \mathrm{meV}$ for Doppler broadening. The lifetime broadening of $\Gamma=675(20) \mathrm{meV}$ agrees well with the value of a single Ar $1 s$ hole (655 meV [21]) but not with the sum of a single Ar $1 s$ hole and a single $2 p$ hole (115 meV [28]). This is due to a decrease of the Ar $1 s$ corehole lifetime broadening due to a decrease of the Auger rates caused by the vacancy in the $2 p$ shell $[29,30]$. Finally, the binding energies are determined from the fit analysis; they are listed in the Supplemental Material [31], together with quantum defects $\delta$ and peak assignments for the $1 s^{-1} 2 p^{-1} n l$ states.

For an initial understanding of the spectrum in Fig. 2, comparison can be made to $\mathrm{Ne} 1 s^{-1} 2 p^{-1} n l$ valence satellites. Assuming that monopole satellite transitions dominate in argon, as they do in neon, one expects the following possibilities [9]:

$$
\begin{aligned}
& \text { (a) } \operatorname{Ar} 1 s^{-1} 2 p^{-1} n p\left({ }^{2} S_{1 / 2}\right) \epsilon p, \\
& \text { (b) } \operatorname{Ar} 1 s^{-1} 2 p^{-1} n s\left({ }^{2} P_{1 / 2,3 / 2}\right) \epsilon s, \epsilon d \text {. }
\end{aligned}
$$

The states of type (a) can be considered as Ar $1 s$ ionization accompanied by shakeup of a $2 p$ core electron into an unoccupied $n p$ Rydberg orbital. In contrast, the (b) states are due to $\operatorname{Ar} 2 p$ ionization accompanied by shakeup of a $1 s$ electron into an unoccupied $n s$ Rydberg orbital. Because a $1 s$ vacancy influences the effective charge seen by the $2 p$ electrons much more than the inverse, it is expected that the (a) states are more intense than the (b) states. This simple picture seems to correspond quite well with the assignments and intensities in the present work.

The bar diagrams in Fig. 2 indicate the energy positions of Rydberg states converging to the ${ }^{3} P_{2,1,0}$ and ${ }^{1} P_{1} \mathrm{DCH}$ thresholds. The clearest series consists of peaks leading to the ${ }^{1} P_{1}$ threshold, with eight distinct features. Based on the simple picture described above, the four most intense peaks are assigned to the $1 s^{-1} 2 p^{-1}\left({ }^{1} P_{1}\right) n p\left({ }^{2} S_{1 / 2}\right)$ Rydberg series, with $n=4-7$. The assignment of this series is confirmed by good agreement with the term values of Ca II [26] (see Supplemental Material [31] for comparison). Using the Rydberg formula for the energies of these four states, the $1 s^{-1} 2 p^{-1}\left({ }^{1} P_{1}\right)$ double-ionization threshold can be determined rather accurately to be $3523.09(5) \mathrm{eV}$. Of the other four peaks below the ${ }^{1} P_{1}$ threshold, the term values for the two at 3511.50 and $3517.97 \mathrm{eV}$ binding energy correspond almost exactly to term values of states in $\mathrm{Ca}$ II, strongly suggesting their assignments as $1 s^{-1} 2 p^{-1}\left({ }^{1} P_{1}\right) n s\left({ }^{2} P_{1 / 2,3 / 2}\right)$, with $n=4,5$, respectively. The two remaining states indicated with ? are very weak, with term values unmatched to $\mathrm{Ca}$ II, suggesting they may be due to nonmonopole transitions. Detailed calculations will be required for a definitive assignment. Below each of the three ${ }^{3} P_{2,1,0}$ DCH thresholds, only two or three Rydberg states are visible due to their overlap and generally lower intensities. However, similar analyses to that used for the ${ }^{1} P_{1}$ states were feasible, and the detailed results are reported in the Supplemental Material [31]. Overall, the DCH thresholds determined in this work lie approximately $2 \mathrm{eV}$ higher than the only existing theoretical values [16]. However, within the error bars, the splittings among the four thresholds agree well with theory.

Comparing the intensities of the $4 p$ and $5 p$ Rydberg states leading to each of the four DCH thresholds, all of which are visible in the spectrum, we find that the intensity ratio between the sum of the 6 peaks below the three ${ }^{3} P$ thresholds and the 2 peaks below the ${ }^{1} P$ threshold is approximately equal to one. This result is quite different from a statistical ratio of three. Preliminary measurements at much higher photon energies, above $7 \mathrm{keV}$, also do not exhibit a statistical ratio. Further studies are warranted to understand this observation.

Together with the above reported detailed description of the nature and energetics of the DCH shake-up states, another significant piece of information can be derived from the present results, and, namely, the 1 s photoabsorption spectrum for an argon ion with a $2 p$ vacancy, as well as a deeper look at the $1 \mathrm{~s}$ photoabsorption curve for the neutral atom. Recently, it has been shown that a photoelectron spectrum of $K^{-2} V$ molecular DCH satellites can be viewed as a NEXAFS spectrum of a molecular ion with a $1 s$ vacancy. However, these results have been obtained by using a multielectron coincidence technique with a detector resolution of only $1.5-2 \mathrm{eV}$ [2], i.e., a value that is much larger than the lifetime broadening for $1 s^{-1} 2 p^{-1}$ states of second-row elements; see above. Moreover, the coincidence technique used in Ref. [2] has very limited abilities to detect high kinetic-energy electrons, which is necessary to use it in the present energy region. In analogy, the sequence of argon Rydberg DCH states shown in Fig. 2, which are dominated by monopole shake-up processes, should correspond to an Ar $1 s$ NEXAFS spectrum taken in the presence of a $2 p$ hole. Thus, the present results illustrate a means to study the spectroscopy of exotic species without the elaborate experimental efforts typically needed to produce highly excited atoms or molecules.

Moreover, in neutral argon, it is well known that lifetime broadening limits the ability to resolve the $n p$ Rydberg series in $1 s$ photoabsorption $[21,32]$. However, two aspects of the present results provide a partial way around this limitation. First, as shown above, the lifetime broadening of an $\operatorname{Ar} 1 s$ hole in a $1 s^{-1} 2 p^{-1} \mathrm{DCH}$ configuration is narrower than for an $\operatorname{Ar} 1 s^{-1}$ single-hole state. Second, due to the Coulomb interaction between the core holes, the energy spacings between adjacent $n p$ Rydberg states in the DCH Rydberg series are larger by approximately a factor of 2.92 compared to the spacings in a conventional Ar $1 s$ photoabsorption spectrum. This factor is not 4 , as one would expect in a one-electron system, because of different values for the quantum defect of the $n p$ series in the $1 s$ absorption 


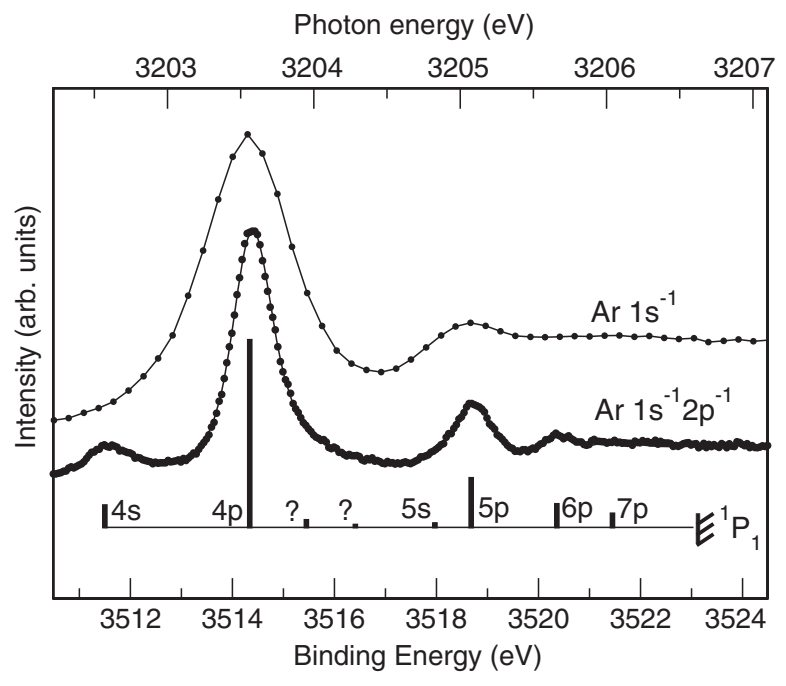

FIG. 3. The Ar $1 s^{-1} 2 p^{-1}\left({ }^{1} P_{1}\right) n l$ shake-up photoelectron spectrum (lower curve, bottom scale) compared to the $\operatorname{Ar} 1 s^{-1}$ photoabsorption spectrum (upper curve, top scale). The energy axis of the $\mathrm{Ar} 1 s^{-1}$ spectrum is stretched by a factor of 2.92 to align the Rydberg series.

spectrum $(\delta \cong 1.8)$ and in the $1 s^{-1} 2 p^{-1}$ shake satellites $(\delta \cong 1.5)$. As an illustration, Fig. 3 compares the $1 s^{-1} 2 p^{-1}\left({ }^{1} P_{1}\right) n l$ DCH satellite spectrum to a standard Ar $1 s$ photoabsorption spectrum, with the energy scale of the latter stretched by 2.92 in order to align the Rydberg series in the spectra. It is immediately evident that the combination of the narrower lifetime width and the larger spacing leads to a better-resolved Rydberg series in the DCH spectrum, including the ability to identify some of the higher members masked by the lifetime broadening in conventional photoabsorption. In addition, the DCH spectrum also exhibits states that are dipole forbidden in photoabsorption. All in all, Fig. 3 demonstrates that the present measurements provide an enhanced view of traditional photoabsorption and a means to partially defeat the lifetime broadening inherent in deep core levels.

In conclusion, states with two core vacancies have been observed in a conventional single-channel photoelectronspectroscopy measurement for the first time, using the argon atom as a prototypical example. A high-quality photoemission spectrum of the $\mathrm{Ar}^{+} 1 s^{-1} 2 p^{-1}(1,3 P) n s, n p$ DCH states was obtained, allowing detailed assignments of the main features, as well as extraction of accurate binding energies, linewidths, and quantum defects. Analysis of the argon DCH photoemission spectrum also confirms that a traditional shake-up picture can be applied to these states in order to provide a qualitative understanding; it is reasonable to consider the $\operatorname{Ar} 1 s^{-1} 2 p^{-1} n s, n p$ states as core-level shake-up satellites of Ar $1 s$ photoionization. In other words, one can think of a photon ionizing a $1 s$ electron while a $2 p$ core electron is excited (shaken up) to an $n p$ Rydberg orbital. Alternatively, one can view the DCH spectrum as akin to $1 s$ photoabsorption by an argon ion with a single $2 p$ vacancy, offering a means to probe photon interactions of such exotic species without the need to create them beforehand. Taking this analogy a step further, this work also demonstrates that the Ar DCH satellite spectrum can be compared to a standard Ar $1 s$ NEXAFS spectrum, thereby improving its effective energy resolution, with the added advantage that the pseudo-NEXAFS spectrum does not require resonant excitation and can be taken at any suitable photon energy. Finally, argon is just a first example of what this new approach can achieve. We have already obtained preliminary results on neon, where we have measured $\mathrm{Ne}$ $K^{-2} V$ DCH shake-up satellites, and in $\mathrm{CS}_{2}$, where we have measured $\mathrm{C} K^{-2} V$ and $\mathrm{S} K^{-2} V$ DCH shake-up states [33]. Double-core-hole states are expected to be ubiquitous throughout the periodic table, as well as in a multitude of molecular species, and we predict a broad range of new studies on atoms, molecules, and other species will soon be available to better understand core-level spectroscopy and dynamics of DCH states, now that this relatively simple experimental approach is available.

Experiments were performed on the GALAXIES beam line at SOLEIL Synchrotron, France. We are grateful to the SOLEIL staff for the smooth operation of the facility. The work at the Freie Universität Berlin was supported by the Deutsche Forschungsgemeinschaft under Project No. PU180/6-1.

[1] J. H. D. Eland, M. Tashiro, P. Linusson, M. Ehara, K. Ueda, and R. Feifel, Phys. Rev. Lett. 105, 213005 (2010).

[2] M. Nakano, P. Selles, P. Lablanquie, Y. Hikosaka, F. Penent, E. Shigemasa, K. Ito, and S. Carniato, Phys. Rev. Lett. 111, 123001 (2013).

[3] P. Lablanquie et al., Phys. Rev. Lett. 107, 193004 (2011).

[4] M. Nakano et al., Phys. Rev. Lett. 110, 163001 (2013).

[5] P. Linusson, S. Fritzsche, J. H. D. Eland, M. Mucke, and R. Feifel, Phys. Rev. A 87, 043409 (2013).

[6] N. Berrah et al., Proc. Natl. Acad. Sci. U.S.A. 108, 16912 (2011).

[7] P. Salén et al., Phys. Rev. Lett. 108, 153003 (2012).

[8] L. S. Cederbaum, F. Tarantelli, A. Sgamellotti, and J. Schirmer, J. Chem. Phys. 85, 6513 (1986).

[9] ESCA Applied to Free Molecules, edited by K. Siegbahn (North-Holland, Amsterdam, 1970).

[10] T. D. Thomas, J. Phys. Chem. A 116, 3856 (2012).

[11] P. H. Kobrin, S. Southworth, C. M. Truesdale, D. W. Lindle, U. Becker, and D. A. Shirley, Phys. Rev. A 29, 194 (1984).

[12] G. B. Armen, T. Åberg, Kh. R. Karim, J. C. Levin, B. Crasemann, G. S. Brown, M. H. Chen, and G. E. Ice, Phys. Rev. Lett. 54, 182 (1985).

[13] S. H. Southworth, T. LeBrun, Y. Azuma, and K. G. Dyall, J. Electron Spectrosc. Relat. Phenom. 94, 33 (1998).

[14] S. Svensson, B. Eriksson, N. Mårtensson, G. Wendin, and U. Gelius, J. Electron Spectrosc. Relat. Phenom. 47, 327 (1988). 
[15] M. Štuhec, A. Kodre, M. Hribar, D. Glavič-Cindro, I. Arčon, and W. Drube, Phys. Rev. A 49, 3104 (1994).

[16] U. Kuetgens and J. Hormes, Phys. Rev. A 44, 264 (1991).

[17] J.-Cl. Dousse and J. Hoszowska, Phys. Rev. A 56, 4517 (1997).

[18] P.-A. Raboud, M. Berset, J.-Cl. Dousse, Y.-P. Maillard, O. Mauron, J. Hoszowska, M. Polasik, and J. Rzadkiewicz, Phys. Rev. A 65, 062503 (2002).

[19] M. Deutsch, N. Maskil, and W. Drube, Phys. Rev. A 46, 3963 (1992).

[20] M. Žitnik, M. Kavčič, K. Bučar, A. Mihelič, and R. Bohinc, J. Phys. Conf. Ser. 488, 012014 (2014).

[21] D. Céolin et al., J. Electron Spectrosc. Relat. Phenom. 190, 188 (2013).

[22] M. Simon et al., Nat. Commun. 5, 4069 (2014).

[23] M. Breinig, M. H. Chen, G. E. Ice, F. Parente, B. Crasemann, and G. S. Brown, Phys. Rev. A 22, 520 (1980).

[24] G. C. King, M. Tronc, F. H. Read, and R. C. Bradford, J. Phys. B 10, 2479 (1977).
[25] L. Avaldi, G. Dawber, R. Camilloni, G. C. King, M. Roper, M. R. F. Siggel, G. Stefani, and M. Zitnik, J. Phys. B 27, 3953 (1994).

[26] NIST Atomic Level Database, Version 5, http://www.nist .gov/pml/data/asd.cfm

[27] G. B. Armen, J. Tulkki, T. Åberg, and B. Crasemann, Phys. Rev. A 36, 5606 (1987).

[28] K. C. Prince, M. Vondráček, J. Karvonen, M. Coreno, R. Camilloni, L. Avaldi, and M. de Simone, J. Electron Spectrosc. Relat. Phenom. 101-103, 141 (1999).

[29] C. P. Bhalla, Phys. Rev. A 8, 2877 (1973).

[30] L. Young et al., Nature (London) 466, 56 (2010).

[31] See Supplemental Material at http://link.aps.org/ supplemental/10.1103/PhysRevLett.114.093001 for the detailed results of the observed resonances.

[32] L. G. Parratt, Phys. Rev. 56, 295 (1939).

[33] R. Püttner et al. (to be published); R. Feifel et al. (to be published). 